\section{Teratogenia da vitamina A}

\section{Vitamin A teratogenicity}

\begin{abstract}
The vitamin A is essential to the preservation and the normal functioning of tissues, as well as, to the growth and development. In the human being it has indirect evidence that the vitamin $A$ in excess, during the first weeks of gestation is teratogenic. Of the opposite, it does not have doubts on the deleterious effect, of a devoid feeding in this micronutrient and on the availability of the knowledge technician to prevent them. The concern with the fact of that the vitamin A would lead it the teratogenicity in human beings, has delayed the implementation of combat programs the vitamin A lack, mainly reaching the programs of food enrichment. Literature is controverted and makes use of few information on the doses for supplementation of pregnant. As retinol circulating maternal is controlled homeostatic after the food consumption vitamin source, it expects after same metabolic reply the strengthened food consumption, indicating that it does not have teratogenicity risk. Consequently, highly seems improbable that the consumption of enriched foods or vitamin supplements the daily pay-formed one, in habitual the single doses, has teratogenic effect in the man.
\end{abstract}

Key words Vitamin A, Teratogens, Vitamin A deficiency

\author{
Maria Helena de Castro Chagas 1 \\ Hernando Flores 2 \\ Florisbela de Arruda Camara e Siqueira Campos 3 \\ Raquel Araújo de Santana 4 \\ Erika Chagas Barreto Lins 5
}

1-5 Departamento de Nutrição. Centro de Ciências da Saúde. Universidade Federal de Pernambuco. Campus Universitário, s/n. Cidade Universitária. Recife, PE, Brasil. CEP: 50.670-901. Email:mhelena@nutricao.ufpe.br

Resumo A vitamina A é essencial à preservação $e$ ao funcionamento normal dos tecidos, assim como, ao crescimento e desenvolvimento. No humano há evidência indireta que a vitamina A em excesso, durante as primeiras semanas de gestação é teratogênica. Do contrário, não há dúvidas sobre os efeitos deletérios, de uma alimentação carente neste micronutriente e sobre a disponibilidade do conhecimento técnico para evitá-los. A preocupação com o fato de que a vitamina A conduziria a teratogenia em humanos, tem retardado a implementação de programas de combate a carência de vitamina $A$, atingindo principalmente os programas de enriquecimento de alimentos. A literatura é controvertida e dispõe de poucas informações sobre as doses para suplementação de gestantes. Como o retinol circulante materno é controlado homeostaticamente após o consumo de alimentos fonte de vitamina $A$, espera-se a mesma resposta metabólica após o consumo de alimentos fortificados, indicando que não há risco de teratogenia. Consequentemente, parece altamente improvável que o consumo de alimentos enriquecidos ou de suplementos de vitamina A pré-formada, nas doses unitárias habituais, tenha efeito teratogênico no homem.

Palavras-chave Vitamina A, Teratogênios, Deficiência de Vitamina A 


\section{Introdução}

A vitamina A, descrita no século passado como fator dietético lipossolúvel $\mathrm{A}^{1,2}$ é um composto que participa de uma série de funções biológicas ${ }^{3}$ e que, por não ser sintetizada no organismo, deve ser fornecido pela dieta. A ingestão insuficiente de vitamina A ou de seus precursores, durante um período expressivo, origina diversas anormalidades, entre as quais, perda de apetite e de peso, alterações epiteliais, diminuição da resistência às infecções, e alterações no ciclo visual. 4

A vitamina A é um álcool (retinol) isoprenóide lipossolúvel e insaturado, encontrado em alimentos de origem animal na forma de ésteres (palmitato). Também está presente em plantas, na forma de carotenóides, precursores de retinol, em especial nos vegetais verdes e folhosos, e vegetais e frutas alaranjadas.

Qualquer que seja a forma do composto ingerido, ele é hidrolisado, para que possa ser absorvido pelas células da mucosa intestinal. Dos carotenóides, o $\beta$-caroteno é o de maior bioconversibilidade, sendo fracionado no citoplasma das células da mucosa intestinal, em duas moléculas de retinoaldeído, que são reduzidas e esterificadas para formar ésteres de retinil.

\section{Aspectos metabólicos}

Na parte superior do intestino delgado o éster é em grande parte hidrolisado liberando o álcool pela ação de uma hidrolase do suco pancreático na presença de sais biliares. Juntamente com os produtos da digestão da gordura, o retinol é emulsionado pelos sais biliares e pelos fosfolipídios, convertendo-se por fim em micelas, adequadas à absorção. Em condições normais, mais de $90 \%$ da vitamina A ingerida é absorvida. ${ }^{4}$

Os ésteres de retinil ao atingirem o lúmem do intestino delgado, sofrem uma hidrólise catalisada pela enzima retinil éster hidrolase, situada na superfície externa da membrana celular da mucosa intestinal. $\mathrm{O}$ retinol resultante desta hidrólise, penetra no interior da célula, onde é reesterificado com ácidos graxos de cadeia longa e é transportado como componente dos quilomícrons, via linfática para rins, músculos e principalmente para o fígado.3,5

Do retinol absorvido, 80 a $90 \%$ são depositados no fígado na forma de éster. Do fígado, o retinil é mobilizado para atender as necessidades do tecido, sendo hidrolisado a retinol.

O retinol circula no plasma ligado a uma proteí- na específica, a Retinol Binding Protein (RBP).

Os quilomícrons remanescentes são captados pelo fígado, e o retinol, cerca de $95 \%$ de vitamina A do organismo, é armazenado nos hepatócitos, sob a forma de palmitato de retinol.3,5

\section{Carência de vitamina A e estratégias de controle}

A deficiência de vitamina A constitui sério problema de saúde pública. O estado carencial pode ser definido por uma série de indicadores bioquímicos e sinais clínicos. Nesse estado, os níveis plasmáticos e hepáticos de vitamina A ficam muito reduzidos e a ingestão de vitamina $\mathrm{A}$ e seus precursores carotenóides é mínima ou nula. 4

A carência de vitamina $A$, em diversos estágios de gravidade, afeta quase um bilhão de pessoas no mundo, principalmente crianças, pré-escolares, grávidas e nutrizes, calculando-se que, a cada ano, esta carência é responsável direta pela morte de mais de dois milhões de crianças nos primeiros anos de vida. 6

Sabe-se que a ingestão de alimentos fontes de vitamina A é inadequada em nosso país. Estima-se que $60 \%$ da população infantil e pré-escolar apresentam níveis de vitamina A circulante abaixo do normal.710 Vários estudos concluem que melhorando o estado nutricional de vitamina A podem ser reduzidas significativamente as taxas de mortalidade infantil e pré-escolar. 11,12

As consequiências da carência de vitamina A são bem conhecidas: retardo do crescimento e desenvolvimento, menor eficiência do sistema imune, cegueira e, como tem sido recentemente demonstrado, maiores índices de morbidade e mortalidade infantil em pré-escolares. ${ }^{13}$

A World Health Organization (WHO), através do International Vitamin A Consultative Group (IVACG) ${ }^{14}$ distingue estratégias para a prevenção e controle da carência.

Sendo a aversão aos principais alimentos fonte de vitamina A praticamente universal 13 entre as crianças, os especialistas propõem três tipos de estratégias: a) suplementação com doses maciças como medida emergencial de curto prazo; b) enriquecimento de alimentos (redistribuição de nutrientes) a médio prazo; c) diversificação dietética como solução definitiva, porém de longo ou muito longo prazo.

Os programas de distribuição de doses maciças costumam obter bons resultados mas com o tempo, tornam-se ineficazes já que dependem essencialmente da vontade política e da participação ativa da 
comunidade.

A diversificação dietética, como solução ideal é difícil e muito demorada pois consiste na mudança de hábitos alimentares e consumo de alimentos de origem vegetal em que a bioconversibilidade do $\beta$ caroteno pode mudar totalmente o enfoque como solução do problema.

Resta o enriquecimento de alimentos, que consiste no aumento do teor de nutrientes num determinado alimento.

Um programa de enriquecimento de alimentos consta de vários elementos essenciais: um alimento vetor (o veículo), o nutriente a ser agregado numa forma que garanta sua estabilidade até chegar ao consumidor, a integridade das características organolépticas do vetor, e uma tecnologia de adição simples e de baixo custo.

Recentemente foi descrita uma pré-mistura que incorpora vitamina A a grãos de arroz reconstituídos. 15 Os testes preliminares em nosso laboratório confirmaram que a vitamina A na pré-mistura é suficientemente estável ao armazenamento e a cocção para quaisquer fins práticos. 16

Esta mistura também demonstrou ser biodisponível, podendo substituir as doses maciças na prevenção e controle da carência de vitamina A.7-10

\section{Teratogenia}

\section{Efeitos teratogênicos no homem}

Há evidências de que a vitamina A em excesso durante as primeiras semanas de gestação é teratogênica em humanos.6,17-20

A ação teratogênica da vitamina $A$ tem sido demonstrada em várias espécies de animais. ${ }^{21} \mathrm{O}$ tipo do defeito no entanto, depende da quantidade de vitamina $\mathrm{A}$, bem como, do estágio gestacional em que a vitamina A é administrada. ${ }^{22}$ As anormalidades encontradas em crianças foram devidas a níveis significativamente altos de retinol no soro de mães após o consumo excessivo de vitamina A.22

Qualquer efeito teratogênico atribuível a ingestão de vitamina A deve estar diretamente relacionado a oscilações na concentração de retinol sérico materno, como acontece com o consumo de uma cápsula de 200.000UI de vitamina A, desde que a vitamina alcance o embrião ou o feto através do sangue materno. Por causa da teratogenicidade da vitamina A em animais e da isotretinoina em humanos, a vitamina $\mathrm{A}$ (não o $\beta$-caroteno) tem sido considerada teratogênica. Há evidências de má-formação em crianças, quando as mães consomem altas doses de vitamina A durante a gestação (>25.000UI/dia). ${ }^{23}$

A Organização Mundial da Saúde, recomendou a redução da exposição de mulheres grávidas a altas doses de vitamina A na forma de suplementos. ${ }^{23}$ As recomendações estão embasadas nos estudos de Mills et al. ${ }^{24}$ e Oakley e Erickson, ${ }^{25}$ que orientam, durante a gestação, o uso de multivitamínicos a base de $\beta$-caroteno, corroborando o recomendado desde 1987 pelo Centro de Controle da Sociedade de Teratogenia. 26

Altas doses de vitamina A têm potente efeito teratogênico em animais, sendo contra-indicadas durante a gravidez. Este alto potencial teratogênico justifica a precaução na indicação da vitamina para mulheres em idade fértil. 27

A captação do retinol pelos tecidos é facilitada pelo complexo RBP - retinol, no entanto este mecanismo pode ser ultrapassado quando altas doses de vitamina A são consumidas. 28

Se o retinol circulante materno for controlado homeostaticamente após o consumo do alimento fortificado com vitamina $\mathrm{A}$, assim como ocorre após o consumo do alimento fonte de vitamina A, como fígado, por exemplo, esta seria uma indicação de que não há risco de teratogenia com alimentos fortificados. 23

Em relação à espécie humana, são raros os dados, sobre uma associação direta entre anomalias do desenvolvimento do embrião e o consumo por gestantes, de doses elevadas de vitamina A pré-formada sob a forma de retinol, ou ésteres de retinil, no início da gravidez. 23

Os efeitos teratogênicos da vitamina A ocorrem pela presença dos metabólitos, ácido transretinóico, ácido 13-cis retinóico e dos seus oxiderivados. Têm sido estudados os casos de mulheres que receberam doses elevadas desses derivados no decorrer das seis primeiras semanas de gestação. ${ }^{23}$ A partir desta idade gestacional não há dados que justifiquem a associação entre a suplementação com vitamina A e teratogenia fetal. ${ }^{23}$

Os estudos experimentais mostram o efeito teratogênico direto do ácido retinóico (trans, 13-cis, 4-oxo-trans, 4-oxo-13-cis) e não do retinol.29-31 Os riscos do aporte excessivo à mãe, referem-se essencialmente aos suplementos que aumentam a concentração de ácido retinóico no soro da mãe, e não aos que aumentam a concentração de retinol ou ésteres de retinil.23

A administração de doses terapêuticas diárias de vitamina A desencadeia picos de concentração sérica de isotretinoina acima de $200 \mathrm{ng} / \mathrm{mL}$ e taxas endógenas entre $1 \mathrm{a} 4 \mathrm{ng} / \mathrm{mL}$.

A isotretinoina é o ponto de origem de um quadro característico de abortos espontâneos, de partos pre- 
maturos e de má-formações, afetando o sistema nervoso central, o desenvolvimento craneo-facial ou o do sistema cardíaco. 23

É geralmente aceito que o retinol e seus metabólitos não têm efeitos teratogênicos em concentrações séricas fisiológicas e que o ácido retinóico desempenha um papel essencial na regulação de numerosos aspectos da embriogênese normal. ${ }^{31}$ Os dados experimentais indicam que a concentração de ácido retinóico no embrião determina, ao menos parcialmente, a especificidade do poder de regulação genética que se atribui ao ácido retinóico. 23

Há evidências de efeitos tóxicos de um consumo excessivo de vitamina A pré-formada para o desenvolvimento do embrião. 24,25 Pelo menos seis relatos de caso, relativamente completos, foram publicados sobre acontecimentos desfavoráveis da gravidez, associados à ingestão diária de 25.000UI de vitamina A pré-formada. Estes relatos destacam um número inesperado de anomalias do aparelho urinário. 23

Estudos de coorte sobre a utilização de vitamina A durante a gestação têm sido apresentados de forma sucinta. $23 \mathrm{Em}$ um, desses estudos, não houve aumento do número de má-formações entre as crianças nascidas de mães que receberam complexo vitamínico contendo 6.000UI de vitamina A pré formada. Outro estudo foi dirigido a um grande coorte de mulheres acompanhadas durante vários anos, e que freqüentavam um centro médico para diagnóstico pré-natal. Em numerosas análises sobre suplementação, o consumo de mais de 10.000 UI de retinol foi considerado como uma forte exposição à vitamina A.28 Dez casos de má formação foram registrados no grupo que recebeu dose mais elevada. Os autores demonstraram que a prevalência das má-formações cranianas entre as crianças nascidas de mães que receberam quantidades superiores a 10.000UI/dia, ultrapassava $1,7 \%$ do total dos nascimentos. 23

Em virtude do efeito teratogênico do ácido retinóico em doses terapêuticas, no homem, parece lógico supor que suplementos de vitamina A pré-formada tenham efeito teratogênico a partir de dosagem ainda desconhecida. $23-25$

Os estudos descritos acima procuram determinar esta dose máxima, qualificando como dose elevada uma quantidade superior a $10.000 \mathrm{UI}$ de vitamina $\mathrm{A}$ pré-formada, por dia.

Estudos recentes parecem indicar que suplementos de até 10.000UI de vitamina A por dia associada a outras vitaminas, reduzem o risco de má-formação quando administrados no período que precede ou sucede a concepção. 25

Embora um bom número de estudos sobre o efeito teratogênico da utilização da vitamina A apre- sentem limitação metodológica, a maioria dos dados disponíveis não permite afirmar que a dose crítica é de $10.000 \mathrm{UI} /$ dia. 23

Parece altamente improvável que o consumo de suplemento de vitamina A pré-formada, nas doses unitárias habituais, tenha efeito teratogênico no homem. 23

\section{Mecanismos de ação teratogênica}

O mecanismo pelo qual a vitamina A exerce efeitos teratogênicos é atribuído à influência das elevadas concentrações de certos metabólitos do ácido retinóico tais como o ácido trans e o 13-cis-retinóico, sobre o funcionamento dos genes em períodos críticos da organogênese e da embriogênese. ${ }^{29}$ Estudos farmacocinéticos realizados em primatas e em mulheres indicam que o risco de efeitos teratogênicos de altas concentrações desses metabólitos, após uma suplementação em dose única, decresce ao fim de cinco dias. 29

Não existem dados para assegurar que a administração de dose única de 100.000 a 200.000UI (30.000 a $60.000 \mu \mathrm{g}$ de éster de retinil), eleve as concentrações de metabólitos do ácido retinóico suscetíveis a efeitos teratogênicos, o tempo em que estas concentrações permanecem elevadas, ou mais exatamente, o momento em que ocorre a exposição a picos de agentes potencialmente teratogênicos. Por isso, é importante esclarecer a farmacocinética dos metabólitos de vitamina A, quando da administração de doses elevadas de ésteres de retinil no início da gravidez, tanto em mulheres que têm reservas elevadas quanto as que apresentam estoques limitados de vitamina A. Por razões éticas, não é possível efetuar estudos prospectivos administrando a gestantes doses suscetíveis de terem efeitos teratogênicos. Estas pesquisas devem então, ser realizadas em mulheres em idade fértil que não corram o risco de engravidar. Pesquisas em voluntários que receberam doses diárias de vitamina A por via oral durante três semanas, mostraram aumento das concentrações sistêmicas de vitamina A e de seus metabólitos após doses de 10.000 e de 30.000UI.23 As doses de 10.000UI provocaram apenas discretas elevações da concentração sistêmica, taxas que não ultrapassaram os limites dos de gestantes sem suplementação durante o primeiro trimestre de sua gestação. As doses de vitamina A não provocaram senão um aumento das taxas séricas que apenas ultrapassaram o espectro das concentrações fisiológicas. 23

O estudo de Rothman et al.28, foi detalhadamente examinado por pesquisadores em virtude da 
controvérsia internacional que provoca. Este estudo mostra que existe um aumento no risco de anomalias congênitas nas mulheres que consomem mais de

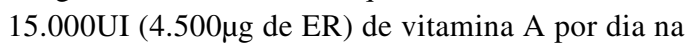

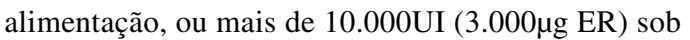
a forma de suplementos. Apesar das críticas pertinentes de Werler et al. 32 publicadas no "New England Journal of Medicine", quanto ao aspecto ético e metodológico, o estudo deve ser examinado em um contexto mais amplo.

\section{Conclusões}

$\mathrm{Na}$ literatura há pouca informação sobre doses semanais ou mensais de vitamina $A$, que representem risco para mulheres em idade fértil ou em diferentes estágios de gestação. Os pesquisadores concluíram que não se poderia por em dúvida a inocuidade de doses diárias de 10.000UI (3.000 $\mu$ g de retinol), sendo recomendadas para mulheres em idade fértil e durante toda a gestação. Consideram ainda menos arriscada a administração de doses superiores a 10.000UI de vita- mina pré-formada ou de ácido retinóico após o primeiro trimestre da gestação. 23

Os efeitos da suplementação podem ser diferentes de acordo com o estado nutricional das mulheres, ou ainda, se as vitaminas forem provenientes de um suplemento concentrado ou de uma fonte alimentar. Os pesquisadores ressaltam que, o risco de concentração elevada de metabólitos no sangue é menor se a fonte de vitamina A for natural (como o fígado animal), do que na forma de suplemento. ${ }^{33}$ Estas conclusões corroboram as recomendações atuais a favor de uma melhoria do estado nutricional de vitamina A das gestantes por meio de regime alimentar.

Com base nos dados da OMS, UNICEF e IVACG, especialistas concluíram que gestantes cujo consumo habitual de vitamina A seja inferior a $800 \mu \mathrm{g}$ de ER não apresentam risco teratogênico com a suplementação de vitamina A pré-formada na dosagem de $10.000 \mathrm{UI}$ ( $3.000 \mu \mathrm{g}$ de retinol). Não recomendam, entretanto, a suplementação diária superior a $8.000 \mathrm{UI}(2.400 \mu \mathrm{g}$ de ER) para aquelas com aportes adequados de vitamina A.23

\section{Referências}

1. McCollum EV, Davis M. The necessity of certain lipids in the diet during growth. J Biol Chem 1917; 15: 167-75.

2. Osborne TB, Mendel LB. The influence of butter-fat on growth. J Biol Chem 1914; 16: 423-37.

3. Blomhoff R, Green MH, Green JB, Berg T, Norum KA. Vitamin A metabolism: new perspectives on absorption, transport and storage. Physiol Rev 1991; 55: 955-8.

4. UNICEF (Fundo das Nações Unidas para a Infância). Carência de vitamina A e xeroftalmia. Brasília (DF): Organização Mundial da Saúde; 1980.

5. Sommer A. Vitamin A supplementation and childhood morbidity. Lancet 1993; 342: 1420-3.

6. Humphrey JH, West KP, Sommer A. Vitamin-A deficiency and attributable mortality among under-5-year-olds. Bul Word Health Organ 1992; 70: 225-32.

7. Campos FACS. Estratégias para a sobrevivência das crianças: arroz enriquecido com vitamina A [tese doutorado]. Recife: Departamento de Nutrição, Centro de Ciências da Saúde da Universidade Federal de Pernambuco; 1999.

8. Flores H, Azevedo MNA, Campos FACS, Barreto-Lins MHC, Cavalcanti AA, Salzano AC, Varela RM, Underwood BA. Serum vitamin A distribution curve for children aged 2-6 y known to have adequate vitamin A status: a reference population. Am J Clin Nutr 1991; 54: 707-11.
9. ICNND (Interdepartmental Committee on Nutrition for National Development, Northeast Brazil). Nutrition survey: 1963. Washington (DC): Government Printing Office; 1965.

10. Santana RA. Eficácia do arroz enriquecido com vitamina A no controle e prevenção da carência de vitamina A [dissertação mestrado]. Recife: Departamento de Nutrição, Centro de Ciências da Saúde da Universidade Federal de Pernambuco; 1996.

11. Barreto ML, Santos LMP, Assis AMO, Araujo MPN, Farenzena GG, Santos PAB, Fiaccone RL. Effect of vitamin A supplementation on diarrhoea and acute lower-respiratory-tract infections in young children in Brazil. Lancet 1994; 23: 228-31.

12. Rosales FJ, Kjolhede C, Goodman S. Efficacy of a single oral dose of 200,000IU of oil-soluble vitamin A in measles-associated morbidity. Am J Epidemiol 1996; 143: 413-22.

13. Flores H, Campos FACS, Silva MBM, Lins MHCB, Barretto EMF. Enriquecimento de alimentos. Cad Cent $\mathrm{Ci}$ Saúde 1995; 4: 1-15.

14. WHO (World Health Organization), IVACG (International Vitamin A Consultative Group). Indicators for assessing vitamin A deficiency and their application in monitoring and evaluating intervention programmes. Geneve: The 
Organization; 1996. (Micronutrient series, WHO/NUT, 10).

15. Murphy P, Smith C, Hauck C, O'Connor K. Stabilization of vitamin A in a synthetic rice premix. J Food Sci 1992; 57: 437-9.

16. Flores H, Guerra NB, Cavalcanti ACA, Campos FACS, Azevedo MCNA, Silva MBM. Bioavailability of vitamin A in a synthetic rice premix. J Food Sci 1994; 59: 371-77.

17. Maden M, Gale E, Zile M. The role of vitamin A in the development of the central nervous system. J Nutr 1998; 128: 471-5.

18. Humphrey JH, Agoestina T, Juliana A, Septiana S, Widjaja H, Cerreto MC, Wu ISF, Ichord RN, Katz J, West KP. Neonatal vitamin A supplementation: effect on development and growth at $3 \mathrm{y}$ of age. Am J Clin Nutr 1998; 68: 109-17.

19. Czeizel A, Rockenbauer M. Prevention of congenital abnormalities by vitamin A. Int J Vit Nutr Res 1998; 68: 219-31.

20. Jick H. Retinoids and teratogenicity. J Am Acad Dermatol 1998; 39: 118-22

21. Hayes WC, Cobel-Geard SR, Hanley TR, MurraY JS, Freshour NL, Rao KS, John JA. Teratogenic effects of vitamin A palmitate in Fischer 344 rats. Drug Chem Toxicol 1981; 4: 283-95.

22. Basu TK. Avitaminosis and congenital malformations. Int $\mathbf{J}$ Vit Nut Res 1983; 24: 9-14.

23. OMS (Organização Mundial da Saúde). Vitamina A na gestação e na lactação: recomendações e relatório de uma consultoria. Recife: A Organização; 2001. (Série Micronutrientes. WHO/NUT/98.4).
24. Mills JL, Simpson JL, Cunningham GC, Conley MR, Rhoads GG. Vitamin A and birth defects. Am J Obstet Gynecol 1997; 177: 31-6.

25. Oakley GP, Erickson JD. Vitamin A and birth defects. N Engl J Med 1995; 333:1414-5.

26. Teratology Society. Position paper: recommendations for vitamin A use during pregnancy. Teratology 1987; 35: 269-75.

27. Guillonneau M, Jacqz Aigrain E. Teratogenicity of vitamin A and derivates. Arch Pediatr 1997; 4: 867-74.

28. Rothman KJ, Moore LL, Singer MR, Nguyen US, Mannino S, Milunsky A. Teratogenicity of high vitamin A intake. N Engl J Med 1995; 333: 1369-73.

29. Tzimas G, Nau H. The role of metabolism and toxicokinetics in retinoid teratogenesis. Cur Pharm Des 2001; 7 803-31.

30. Lewis KC, Green MH, Green JB, Zech LA. Retinol metabolism in rats with low vitamin A status: a compartmental model. J Lipid Res 1990, 31: 1535-48.

31. Azais-Braesco V, Pascal G. Vitamin A in pregnancy: requirements and safety limits. Am J Clin Nutr 2000; 71 : 1325-33.

32. Werler MM, Lammer EJ, Mitchell AA. Teratogenicity of high vitamin A intake. N Eng J Med 1996; 334: 1195-7.

33. Buss NE, Tembe EA, Prendergast BD, Renwick AG, George CF. The teratogenic metabolites of vitamin A in women following supplements and liver. Hum Exp Toxicol 1994; 13: 33-43.

Recebido em 3 de fevereiro de 2003

Versão final reapresentada em 29 de abril de 2003

Aprovado em 30 de junho de 2003 